\title{
TiCrVMo alloys with high dissociation pressure for high-pressure MH tank
}

\author{
T. Matsunaga ${ }^{a, b, *}$, M. Kon ${ }^{a}, K$. Washio ${ }^{a}$, T. Shinozawa ${ }^{a}$, M. Ishikiriyama ${ }^{a}$ \\ ${ }^{a}$ Advanced Material Engineering Division, Higashifuji Technical Center, Toyota Motor Corporation, 1200 Mishuku, \\ Susono, Shizuoka, 410-1193 Japan \\ bPhysics Department, University of Fribourg, Pérolles, 1700 Fribourg, Switzerland
}

\begin{abstract}
High-pressure metal hydride (MH) tank is a possible hydrogen storage system for fuel cell vehicles. The merit of the high-pressure MH tank system is improved by the use of a metal hydride with high dissociation pressure. In this study, TiCrV and TiCrVMo alloys with BCC structure has been developed for the high-pressure $\mathrm{MH}$ tank system. The developed TiCrVMo alloy shows 2.4 mass\% of effective hydrogen capacity between $0.1 \mathrm{MPa}$ and $33 \mathrm{MPa}$ at $298 \mathrm{~K}$, which has a dissociation pressure of $2.3 \mathrm{MPa}$ at $298 \mathrm{~K}$. By investigating the dissociation pressures of the synthesized metal hydrides, it is found that Mo has a special effect to increase dissociation pressure of the metal hydrides. This effect is probably attributed to the large bulk modulus of Mo compared to other elements.
\end{abstract}

\section{Introduction}

Metal hydride is one of the most promising candidates for hydrogen storage materials for fuel cell vehicle because of its high gravimetric density [1]. Recently, high-pressure metal hydride $(\mathrm{MH})$ tank has been reported as a possible hydrogen storage system for fuel cell vehicles [2-4]. Fig. 1 shows a schematic view of a high-pressure $\mathrm{MH}$ tank. In many cases, as hydrogen storage materials are in powder forms, the packing densities of the materials are limited. Therefore, more than $50 \%$ of the inner volume of the tank remains empty even when the tank is filled with the maximum amount of the alloy. At that time, by filling this empty space with high-pressure hydrogen gas, volumetric hydrogen storage density as a whole tank system can be improved considerably. Mori et al. have reported that $35 \mathrm{MPa}$ of high pressure compressed gas tank combined with heat exchanger and metal hydride (TiCrMn: effective $\mathrm{H}_{2}$ capacity:1.9 mass\% [5]) can store more than twice the amount of hydrogen as compared to a normal $35 \mathrm{MPa}$ compressed gas tank at the same volume [3]. However, due to the heavy weight of metal hydride, high-pressure metal hydride tank is so heavy that further improvement of gravimetric hydrogen storage capacity is expected.

The merit of a high-pressure $\mathrm{MH}$ tank system is improved by the use of a metal hydride with high dissociation pressure. It is important for a hydrogen storage tank of a fuel cell vehicle to supply hydrogen even at low temperatures. Using a metal hydride with high dissociation pressure, hydrogen can be easily supplied even at low temperatures. Moreover, there is another merit for using a metal hydride with high dissociation pressure from the viewpoint of heat exchange. By increasing the dissociation pressure of a metal hydride, reaction heat

\footnotetext{
* Corresponding author. Advanced Material Engineering Division, Higashifuji Technical Center, Toyota Motor Corporation, $1200 \mathrm{Mishuku,}$ Susono, Shizuoka, 410-1193 Japan. Tel.: +81 55997 7837; fax: +81 559977879.

E-mail address: tomoya@matsunaga.tec.toyota.co.jp (T. Matsunaga).
} 


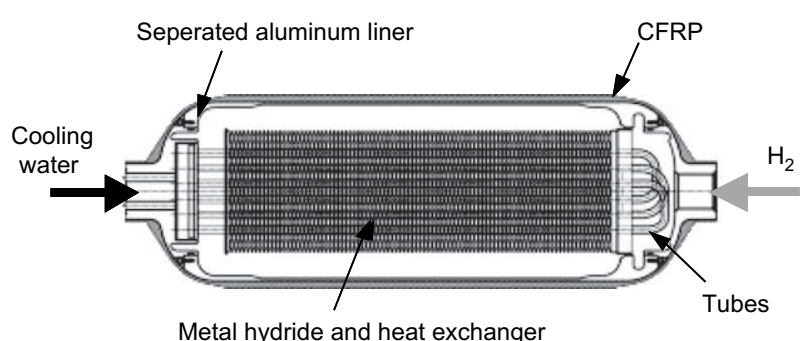

Fig. 1 - A schematic view of a high-pressure MH tank.

during hydrogen desorption $(\Delta H)$ is decreased, which makes heat exchange easier in charging and discharging of hydrogen $[3,4]$. Therefore, besides the hydrogen storage capacity, the dissociation pressure of a metal hydride is also one of the most important factors to apply for a metal hydride tank system.

TiCrV alloy with BCC structure has been studied for years as a promising hydrogen storage material [6-15]. It has two plateau pressures, where only the upper plateau pressure area can be used for reversible hydrogen storage in normal condition. Arashima et al. have reported the alloy with 2.8 mass $\%$ of reversible hydrogen capacity [8]. However, as most of the previous works on these alloys have been aimed for low pressure (less than $1 \mathrm{MPa}$ ) system, dissociation pressures of these materials are often not enough to be applied for the high-pressure $\mathrm{MH}$ tank system. In this study, TiCrV and TiCrVMo alloys with BCC structure are developed in order to apply for high-pressure $\mathrm{MH}$ tank system. For this purpose, dissociation pressure of the metal hydrides is one of the most important parameters of the material. Therefore, by investigating the dissociation pressure of each metal hydride, major factors which influence on those dissociation pressures will be also discussed.

\section{Experimental}

Several compositions of TiCrV and TiCrVMo alloys were prepared by arc melting from pure $\mathrm{Ti}, \mathrm{Cr}, \mathrm{V}$ and Mo elements. The compositions of the alloys are $\mathrm{Ti}_{a} \mathrm{Cr}_{b} \mathrm{~V}_{c} \mathrm{Mo}_{d}(a=14-45$, $b=14-55, \quad c=0-50, \quad d=0-30 \quad[\mathrm{~mol} \%], \quad a+b+c+d=100)$. Subsequently, the alloys were kept at $1473 \mathrm{~K}$ for $2 \mathrm{~h}$ in Argon gas. After heat treatment, the alloys were cooled down slowly

Table 1 - Compositions and lattice constants of the synthesized ternary TiCrV alloys.

\begin{tabular}{|c|c|c|c|c|}
\hline \multirow[t]{2}{*}{ Sample no. } & \multicolumn{3}{|c|}{ Composition [mol\%] } & \multirow{2}{*}{$\begin{array}{c}\text { Lattice constant } \\
\text { [Angstrom] }\end{array}$} \\
\hline & $\mathrm{Ti}$ & $\mathrm{Cr}$ & $\mathrm{V}$ & \\
\hline 1 & 15 & 40 & 45 & 2.995 \\
\hline 2 & 17 & 48 & 35 & 2.997 \\
\hline 3 & 17 & 43 & 40 & 3.001 \\
\hline 4 & 20 & 45 & 35 & 3.006 \\
\hline 5 & 25 & 50 & 25 & 3.010 \\
\hline 6 & 25 & 40 & 35 & 3.026 \\
\hline
\end{tabular}

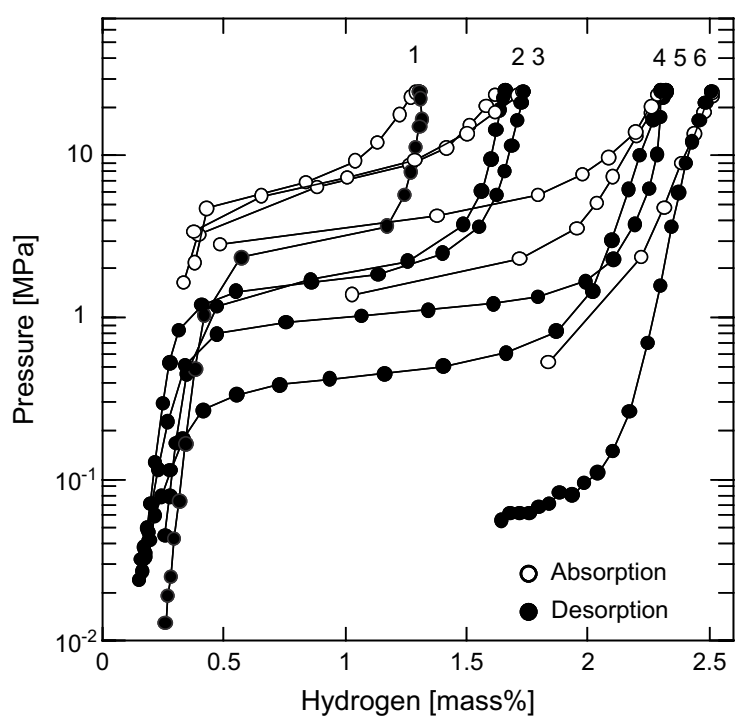

Fig. 2 - PC isotherms of TiCrV alloys at $298 \mathrm{~K}$. The numbers (1-6) correspond to the same sample numbers in Table 1.

to room temperature and then crushed into particles whose diameters were around 0.5-1.0 mm.

Hydrogen storage properties of the materials were investigated using a specially designed Sievelt's type apparatus (maximum pressure: $33 \mathrm{MPa}$ ). $10 \mathrm{~g}$ of the samples were used for each measurement. Before hydrogen absorption measurement, the samples were heated up to $573 \mathrm{~K}$ in vacuum and then cooled down to room temperature. After this pretreatment, hydrogen gas was charged up to $33 \mathrm{MPa}$ at $298 \mathrm{~K}$. Effective hydrogen capacity was measured as the desorbed hydrogen amount at the 1st cycle in the pressure range of 0.1-33 MPa. PC isotherm of each material was measured at $298 \mathrm{~K}$ by the 2 nd absorption-desorption cycle. Dissociation pressure of each hydride is decided as the pressure at the center of the plateau of hydrogen desorption.

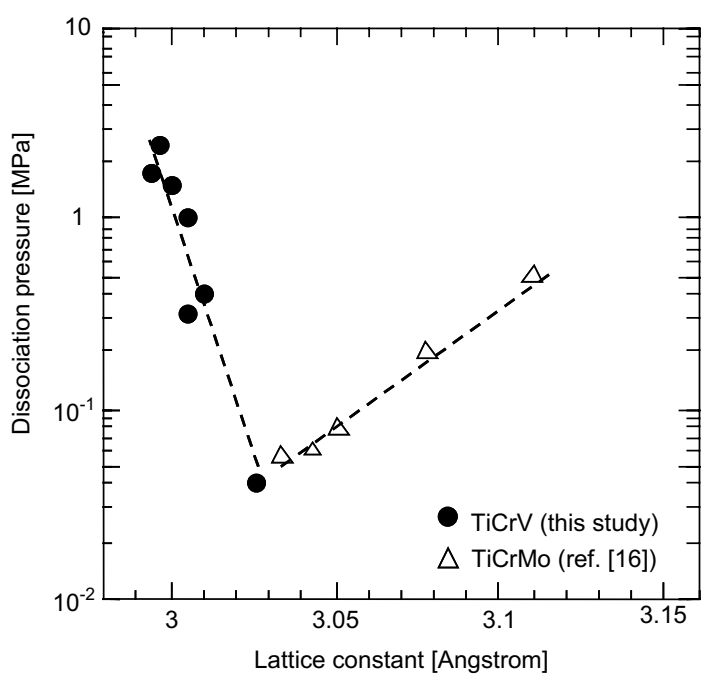

Fig. 3 - Correlation of lattice constants and dissociation pressures in TiCrV and TiCrMo. 

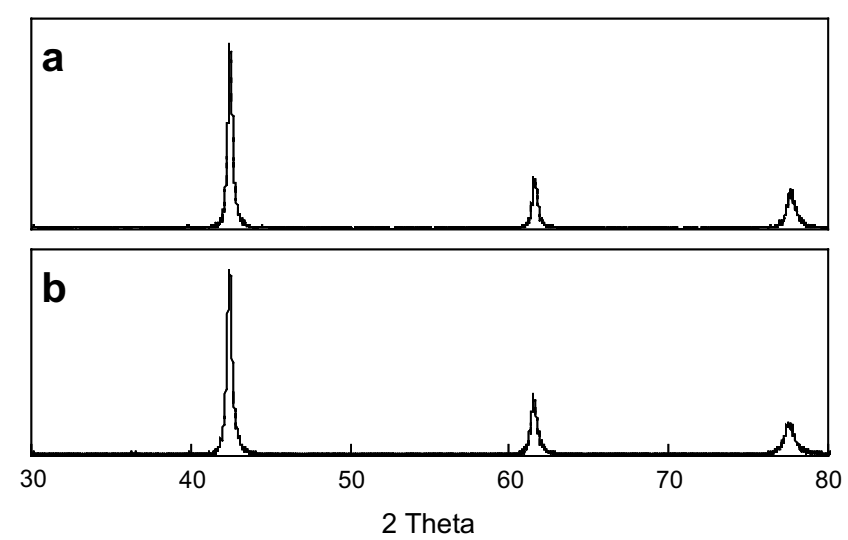

Fig. 4 - XRD profiles of $\mathrm{Ti}_{25} \mathrm{Cr}_{50} \mathrm{~V}_{25}$ and $\mathrm{Ti}_{25} \mathrm{Cr}_{50} \mathrm{~V}_{20} \mathrm{Mo}_{5}$.

Crystal structures and lattice constants of the alloys after the heat treatment were examined by powder X-ray diffraction analysis with $\mathrm{Cu}$ Ka radiation using a Rigaku RINT2500V diffractometer.

\section{Results and discussion}

\subsection{TiCrV alloy}

Six compositions of TiCrV ternary alloys were synthesized as is shown in Table 1. After the heat treatment, all of the alloys were found to be BCC single phase by X-ray diffraction analysis. The lattice constants of them are shown in Table 1. PC isotherms at $298 \mathrm{~K}$ of the alloys are shown in Fig. 2. Dissociation pressure of the alloy increases with the decrease of the lattice constant. This trend is consistent with a general one often observed for other metal hydride systems. However, the effective hydrogen capacity decreases with the increase of the lattice constant. The maximum effective hydrogen capacity is obtained with $\mathrm{Ti}_{25} \mathrm{Cr}_{50} \mathrm{~V}_{25}$, which shows 2.4 mass $\%$ with the

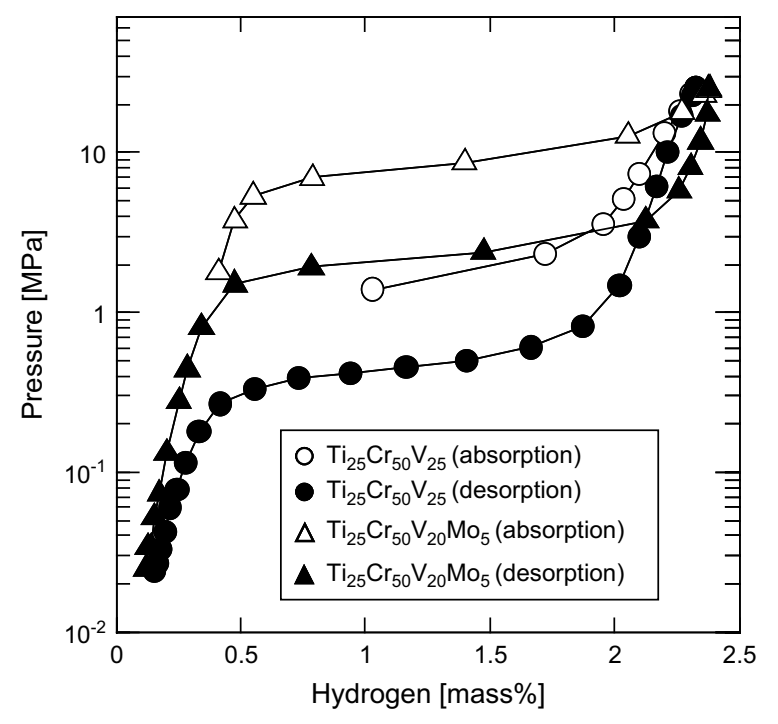

Fig. 5 - PC isotherms of TiCrV and TiCrVMo.
Table 2 - Lattice constants changed by substituting $5 \mathrm{~mol} \%$ of Mo for $\mathrm{V}$.

\begin{tabular}{lccccc} 
Sample no. & \multicolumn{3}{c}{ Composition [mol\%] } & Lattice constant \\
\cline { 2 - 4 } & $\mathrm{Ti}$ & $\mathrm{Cr}$ & $\mathrm{V}$ & $\mathrm{Mo}$ & \\
\hline [Angstrom]
\end{tabular}

dissociation pressure of $0.4 \mathrm{MPa}$ at $298 \mathrm{~K}$. As a result, in the synthesized TiCrV ternary alloys, both high effective hydrogen capacity and high dissociation pressure (more than $1 \mathrm{MPa}$ ) are not satisfied simultaneously.

\subsection{TiCrVMo alloy}

Fig. 3 shows a correlation of dissociation pressures and lattice constants for TiCrV in this study and for TiCrMo from Ref. [16]. Both of TiCrV and TiCrMo are solid solutions consisting of BCC phase. However, in TiCrMo, the correlation of dissociation pressure and lattice constant has a different trend from TiCrV [16]. Kubo et al. have also reported the same trend for TiCrMo alloys [13]. These studies imply that Mo has an effect to increase the dissociation pressure of the metal hydride. In order to confirm this assumption, the alloys which $5 \mathrm{~mol} \%$ of Mo was substituted for $\mathrm{V}$ from the TiCrV alloys were synthesized. After the heat treatment, all the alloys were found to be BCC single phase just like TiCrV ternary alloys. For example, X-ray diffraction data of $\mathrm{Ti}_{25} \mathrm{Cr}_{50} \mathrm{~V}_{25}$ and $\mathrm{Ti}_{25} \mathrm{Cr}_{50} \mathrm{~V}_{20} \mathrm{Mo}_{5}$ are shown in Fig. 4. PC isotherms of $\mathrm{Ti}_{25} \mathrm{Cr}_{50} \mathrm{~V}_{25}$ and $\mathrm{Ti}_{25} \mathrm{Cr}_{50} \mathrm{~V}_{20} \mathrm{Mo}_{5}$ at $298 \mathrm{~K}$ are shown in Fig. 5. By substituting $5 \mathrm{~mol} \%$ of Mo for V, the dissociation pressure increased from $0.4 \mathrm{MPa}$ to $2.3 \mathrm{MPa}$. Note that at that time effective hydrogen capacity did not decrease (2.4 mass\%).

To examine the reason for this result, the lattice constants of the Mo substituted alloys were investigated as is shown in Table 2. Fig. 6 shows a correlation of dissociation pressures

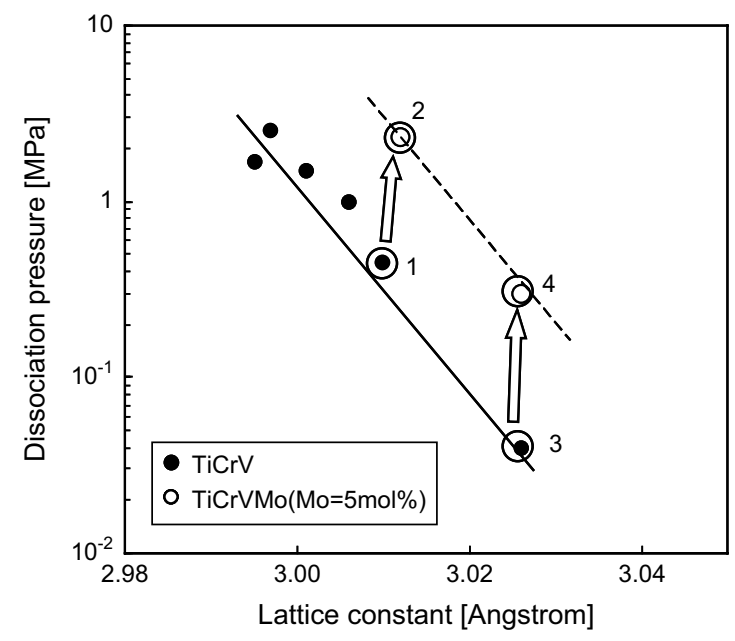

Fig. 6 - Correlation of lattice constant and dissociation pressure of TiCrV and TiCrVMo. 


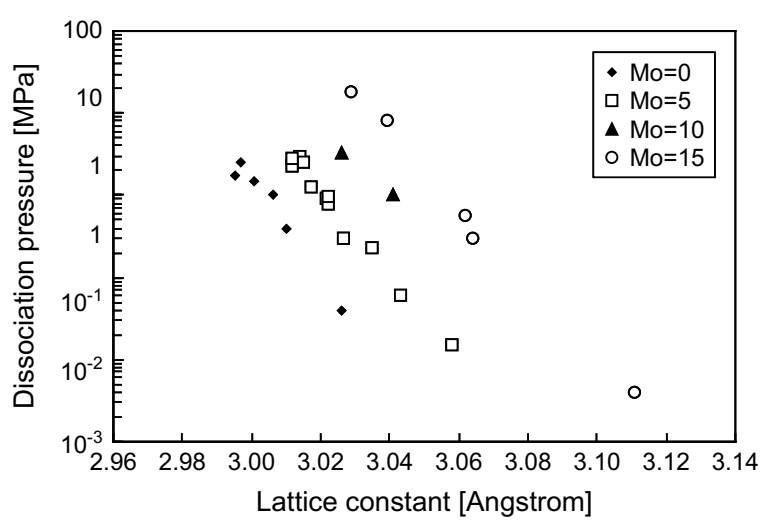

Fig. 7 - Correlation of lattice constant and dissociation pressure of TiCrMoV for various Mo contents.

and lattice constants in TiCrV and TiCrVMo alloys synthesized in this study. By substituting $5 \mathrm{~mol} \%$ of Mo for $\mathrm{V}$, lattice constant does not change. This is probably because the atomic radius of Mo is similar to that of $\mathrm{V}$ (the atomic radius of $\mathrm{V}$ is $1.32 \AA$, where that of Mo is $1.36 \AA$ ). However, at that time, dissociation pressure increases drastically. Fig. 7 shows the correlation of dissociation pressures and lattice constants for various Mo contents (Mo $=0,5,10,15$ [mol\%]). When Mo content is constant, the dissociation pressures seem to have a good correlation with the lattice constants of the alloys. In addition, it can be mentioned that the dissociation pressure also changes according to Mo content of the alloys. This result indicates that Mo has a special effect on increasing the dissociation pressure of the metal hydride.

\subsection{An explanation for Mo effect}

In order to clarify the reason for the Mo effect, the relationship of the dissociation pressures with bulk moduli of the alloys were investigated. Nagasako et al. have confirmed by firstprinciples calculation that the dissociation pressure of a metal hydride consisting of transition metals is highly influenced by

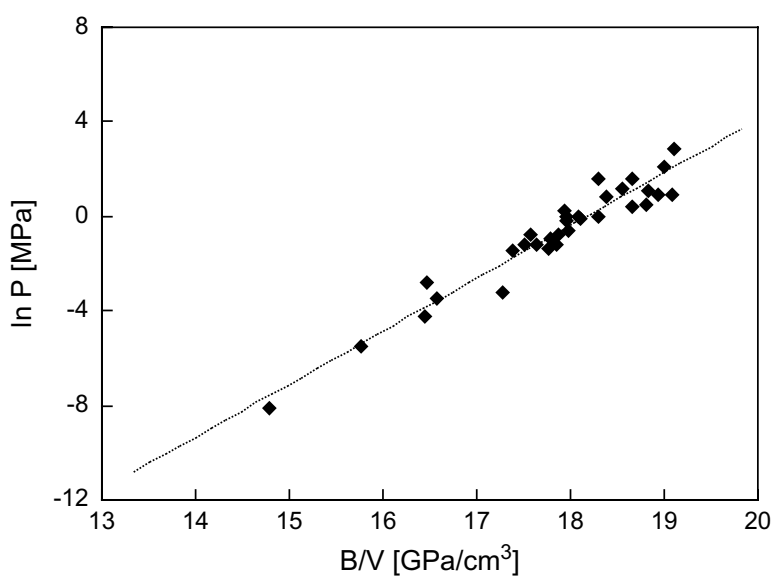

Fig. 8 - Dissociation pressure and B/V for TiCrVMo alloys synthesized in this study for various Mo contents (Mo = 0-15).

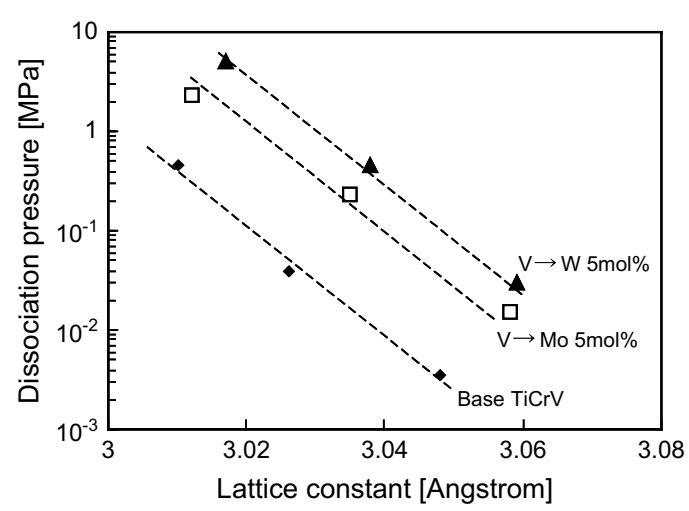

Fig. 9 - Lattice constant and dissociation pressure of TiCrV, TiCrVMo (Mo $=5 \mathrm{~mol} \%$ ) and TiCrVW (W = $5 \mathrm{~mol} \%$ ).

its bulk modulus [17]. This trend has been also experimentally confirmed by Kojima et al. for TiCrMn alloys with Laves phase [5]. According to the results reported by Nagasako et al., there is a linear correlation between the reaction heat $(\Delta H)$ for the hydrogen desorption of a metal hydride and $B / V$, where $B$ and $\mathrm{V}$ are the bulk modulus and the equilibrium volume of the host alloy, respectively. Under the condition that temperature and entropy change of the reaction are constant, the dissociation pressure $(P)$ can be described as follows.

$\ln P \propto \frac{B}{V}$

Fig. 8 shows a correlation of $\ln P$ and $B / V$ for TiCrVMo alloys synthesized in this study for various Mo contents. One good linear correlation can be observed between these two parameters for all the compositions. This result indicates that the Equation (1) can be also applied for TiCrVMo alloys with BCC structure. Therefore, the reason for the Mo effect on the dissociation pressure is probably due to the higher bulk Modulus of Mo compared to other consisting elements ( $\mathrm{Ti}=105.1, \mathrm{Cr}=190.1, \mathrm{~V}=161.9, \mathrm{Mo}=272.5$ [GPa]) [18].

Tungsten has a larger bulk modulus compared to Molybdenum (W=323.2 [GPa]), which implies that Tungsten has a larger effect to increase the dissociation pressure compared to Molybdenum. Fig. 9 shows a correlation between lattice constants and dissociation pressures for TiCrV, TiCrVMo (Mo $=5 \mathrm{~mol} \%$ ) and TiCrVW (W=5 mol\%). It can be clearly observed that Tungsten has a higher effect to increase dissociation pressure. This result also supports the assumption above.

\section{Conclusions}

TiCrV and TiCrVMo alloys with BCC structure have been developed for high-pressure $\mathrm{MH}$ tank system. The developed TiCrVMo alloy shows 2.4 mass $\%$ of effective hydrogen capacity in the pressure range between $0.1 \mathrm{MPa}$ and $33 \mathrm{MPa}$ at $298 \mathrm{~K}$. In TiCrV, the dissociation pressure of the alloy increases with the decrease of the lattice size. This trend is consistent with a general one often observed for other metal hydride systems. However, for TiCrVMo alloy, the dissociation pressure 
is sensitive not only to the lattice size but also to the content of Mo. This indicates that Mo has a special effect to increase the dissociation pressure of the hydride. As a result, it turned out that this effect is probably attributed to the large bulk modulus of Mo compared to other elements. Using this correlation, the dissociation pressure of a metal hydride can be estimated, which will help designing a new material with moderate stability.

\section{Acknowledgements}

The authors would like to thank Prof. Andreas Züttel of EMPA, Dept. Environment, Energy and Mobility, Abt. 138 "Hydrogen \& Energy" for valuable discussions.

\section{R E F E R E N C E S}

[1] Schlapbach L, Züttel A. Hydogen-storage materials for mobile applications. Nature 2001;414:353.

[2] Takeichi N, Senoh H, Yokota T, Tsuruta H, Hamada K, Takeshita HT, et al. "Hybrid hydrogen storage vessel", a novel high-pressure hydrogen storage vessel combined with hydrogen storage material. Int J Hydrogen Energy 2003; 28:1121.

[3] Mori D, Haraikawa N, Kobayashi N, Kubo H, Toh K, Tsuzuki $\mathrm{M}$, et al. High-pressure metal hydride tank for fuel cell vehicles. Mater Res Soc Symp Proc 884E, GG6.4.1.

[4] Mori D, Hirose K, Haraikawa N, Takiguchi T, Shinozawa T, Matsunaga $T$, et al. High-pressure metal hydride tank for fuel cell vehicles. JSAE 20077268/SAE 2007-01-2011. $560-4$.

[5] Kojima Y, Kawai Y, Towata S, Matsunaga T, Shinozawa T, Kimbara M. Development of metal hydride with high dissociation pressure. J Alloys Compd 2006;419:256.
[6] Akiba E, Okada M. Metallic hydrides III: body-centered-cubic solid-solution alloys. MRS Bull 2002;27:699.

[7] Tamura T, Kazumi T, Kamegawa A, Takamura H, Okada M. Protium absorption properties and protide formations of Ti-Cr-V alloys. J Alloys Compd 2003;356-357:505-9.

[8] Arashima H, Takahashi F, Ebisawa T, Itoh $\mathrm{H}$, Kabutomori T. Correlation between hydrogen absorption properties and homogeneity of $\mathrm{Ti}-\mathrm{Cr}-\mathrm{V}$ alloys. J Alloys Compd 2003;356-357:405.

[9] Seo CY, Kim JH, Lee PS, Lee JY. Hydrogen storage properties of vanadium-based b.c.c. solid solution metal hydrides. J Alloys Compd 2003;348:252-7.

[10] dos Santos DS, Bououdina M, Fruchart D. Structural and thermodynamic properties of the pseudo-binary $\mathrm{TiCr}_{2-\mathrm{x}} \mathrm{V}_{\mathrm{x}}$ compounds with $0.0 \leq x \leq 1.2$. J Alloys Compd 2002;340(1-2): 101-7.

[11] Mazzolai G, Coluzzi B, Biscarini A, Mazzolai FM, Tuissi A, Agresti $F$, et al. Hydrogen-storage capacities and $\mathrm{H}$ diffusion in bcc TiVCr alloys. J Alloys Compd 2008;466(1-2):133-9.

[12] Santos SF, Hout J. Hydrogen storage in $\operatorname{TiCr}_{1.2}(\mathrm{FeV})_{x}$ BCC solid solutions. J Alloys Compd 2008. doi:10.1016/j.jallcom.2008.04. 062.

[13] Kubo K, Itoh $\mathrm{H}$, Takahashi $\mathrm{T}$, Ebisawa $\mathrm{T}$, Kabutomori $\mathrm{T}$, Nakamura $\mathrm{Y}$, et al. Hydrogen absorbing properties and structures of Ti-Cr-Mo alloys. J Alloys Compd 2003;356-357: 452-5.

[14] Singh BK, Shim G, Cho S-W. Effects of mechanical milling on hydrogen storage properties of $\mathrm{Ti}_{0.32} \mathrm{Cr}_{0.43} \mathrm{~V}_{0.25}$ alloy. Int $\mathrm{J}$ Hydrogen Energy 2007;32:4961-5.

[15] Lin HC, Lin KM, Wu KC, Hsiung HH, Tsai HK. Cyclic hydrogen absorption-desorption characteristics of $\mathrm{TiCrV}$ and $\mathrm{Ti}_{0.8} \mathrm{Cr}_{1}$. ${ }_{2}$ V alloys. Int J Hydrogen Energy 2007;32:4966-72.

[16] Kamegawa A, Tamura T, Takamura H, Okada M. Protium absorption-desorption properties of Ti-Cr-Mo bcc solid solution alloys. J Alloys Compd 2003;356-357:447.

[17] Nagasako N, Fukumoto A, Miwa K. First-principles calculations of C14-type Laves phase Ti-Mn hydrides. Phys Rev B 2002;66:155106.

[18] Kittel C. Introduction to solid state physics. 7th ed. New York, USA: John Wiley \& Sons. 